\title{
Management for Pediatric Pleural Empyema in Resource-Poor Country: Is Chest Tube Drainage with Antiseptic Lavage-Irrigation Better than Tube Thoracostomy Alone?
}

\author{
${ }^{1}$ Thoracic Surgery Department, Hospital of Mali, Bamako, Mali \\ ${ }^{2} 23^{\text {ème }}$ Chinese Aids Medical Team, Hospital of Mali, Bamako, Mali \\ ${ }^{3}$ Surgery "A" Department, Hospital of Point G, Bamako, Mali \\ Email: "drseydoutg@hotmail.fr
}

Seydou Togo ${ }^{1^{*}}$, Moussa Abdoulaye Ouattara1, Ibrahim Sangaré1, Jacque Saye ${ }^{1}$, Cheik Amed Sékou Touré1, Ibrahim Boubacar Maiga ${ }^{1}$, Dokore Jerome Dakouo', Liang Guo², Sékou Koumaré3, Adama Konoba Koita ${ }^{3}$, Zimogo Zié Sanogo ${ }^{3}$, Sadio Yéna1

Received 25 November 2015; accepted 25 December 2015; published 28 December 2015

Copyright (C) 2015 by authors and Scientific Research Publishing Inc.

This work is licensed under the Creative Commons Attribution International License (CC BY). http://creativecommons.org/licenses/by/4.0/

(c) (i) Open Access

\section{Abstract}

Background: Drainage by chest tube thoracostomy is widely used in treatment of early empyema thoracis in children, but drainage with antiseptic lavage-irrigation is more frequent in our context since the last 20 years. This study was to determine which was more effective in our experience comparing chest tube drainage with catheter antiseptic lavage-irrigation versus drainage by chest tube thoracostomy alone in the management of empyema thoracis in children. Patients and Methods: Demographic, clinical and microbiological data on children with thoracic empyema undergoing drainage by chest tube thoracostomy alone or with antiseptic lavage-irrigation were obtained from 2 thoracic surgical centers from September 2008 to December 2014. It was a retrospective study included 246 children (137 boys and 109 girls) who were managed for empyema thoracis at the author's different department of surgery. Outcomes analysis with respect to treatment efficacy, hospital duration, chest tube duration, hospital costs, and need for subsequent procedures was analyzed and compared in the 2 groups. Results: Drainage of pus and antiseptic irrigation resulted in resolution of pyrexia with improvement in general condition in $85.82 \%$ of patients in group 1 and by tube thoracostomy alone in $73.95 \%$ in group 2 . There are a significant difference in the length of hospital stay $(p=0.022)$, duration of chest tubes in situ $(p=0.040)$, treatment coast $(p=0.015)$ and outcome of stage 2 empyema disease $(p=0.037)$ between the 2 groups. Conclusion: it seems that chest tube drainage with antiseptic lavage-irrigation method is associated with a higher efficacy, shorter length of hospital stay, shorter duration of chest tube in

${ }^{*}$ Corresponding author.

How to cite this paper: Togo, S., et al. (2015) Management for Pediatric Pleural Empyema in Resource-Poor Country: Is Chest Tube Drainage with Antiseptic Lavage-Irrigation Better than Tube Thoracostomy Alone? Surgical Science, 6, 541-548. http://dx.doi.org/10.4236/ss.2015.612077 
situ, less cost and better outcome of stage 2 empyema diseases than a treatment strategy that utilizes chest tube thoracostomy alone.

\section{Keywords}

\section{Empyema, Thoracis, Children, Irrigation, Drainage}

\section{Introduction}

Childreen empyema thoracis is an acute illness associated with significant morbidity, including persistent fever and prolonged hospital stay [1] [2]. Pleural empyema rarely complicates community acquired pneumonia, but its incidence seems to have increased in children [3]. Despite the advances in antibiotic therapy during the last decades, thoracic empyema remains a common clinical entity with high mortality ( $6 \%-24 \%)$ due to delay in initiation of proper treatment [1]. Rapid diagnosis of the causal agent of empyema is required for effective treatment and a good outcome [4]. The decision of the different types of treatments for different stages of the children thoracic empyema is usually empiric, sometimes guided by the failure of treatment modality [5]. Drainage of pus associated with antiseptic lavage-irrigation resulted in resolution of pyrexia with improvement in general condition in patients. We compared Chest tube drainage with antiseptic lavage-irrigation versus drainage by tube thoracostomy alone in management option for empyema thoracis in children and share our experience in resource-poor country with the literature review.

\section{Patients and Methods}

We conducted a prospective study over a 6-year period, from January 2008 to December 2014, at 2 thoracic surgical centers. It was a retrospective study included 246 children (137 boys and 109 girls) who were admitted and managed for empyema thoracis at the author's different department of surgery. All the patients presented with complaints of cough, fever and breathlessness of variable durations. This study included all children (age, 1 to 12 years) admitted to the differents institutions because of community-acquired pleural empyema. 152 patients were 1 to 5 years and 94 patients were 6 to 12 years of age. Neonatology patients and patients with empyema associated with tuberculosis or malignancy were excluded.

Patients were divided in 2 groups with 1:1 trial. Patients for group 1 represented 127 children and underwent treatment by chest tube drainage with chest catheter antiseptic lavage-irrigation; patients for group 2 represented 119 children and underwent drainage by chest tube thoracostomy alone (Figure 1). The technique was well tolerated in all cases. The intrapleural fibrinolytics are not been used in our patients.
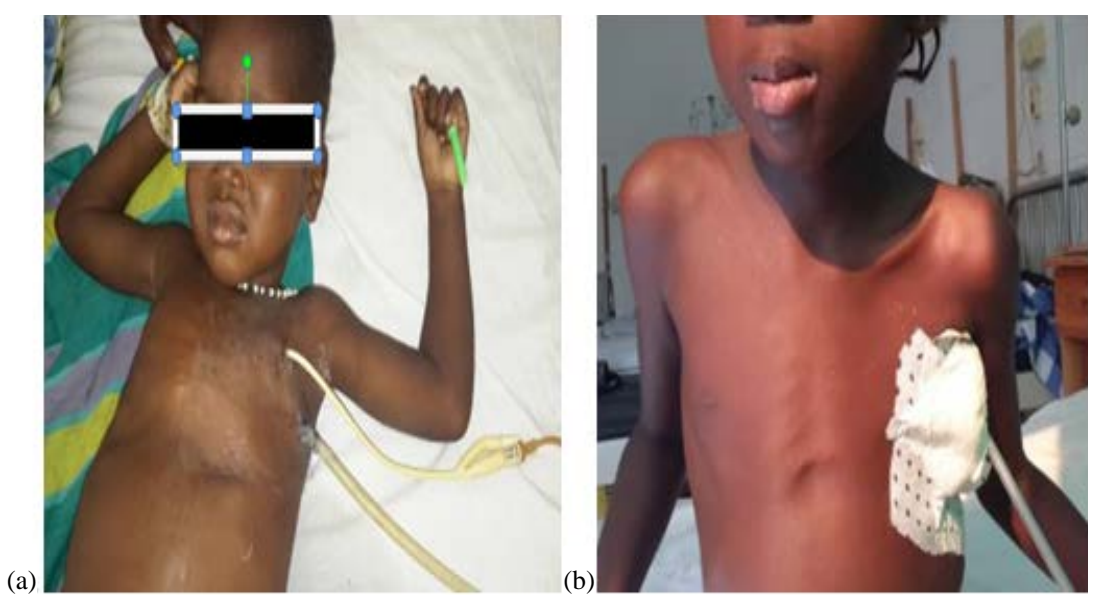

Figure 1. Different methods of drainage in children thoracic empyema: (a) drainage with antiseptic lavage-irrigation; (b) drainage by tube thoracostomy alone. 
Catheter for perfusion or urinary catheter were usually placed in the $2^{\text {nd }}$ intercostal space at the anterior medio-clavicular line and used for the antiseptic lavage-irrigation. The chest tube thoracostomy was placed in the $5^{\text {th }}$ or $6^{\text {th }}$ intercostals space at lateral medio-axillary line. For loculated pleural empyema, lavage-irrigation catheter was placed in the same site with the chest tube after chest echography performed to determine the location of the empyema. For the patients of group 1, the common antiseptic we used was the povidone iodine (Betadin ${ }^{\circledR}$ ) $5 \mathrm{ml}$ in $500 \mathrm{ml}$ of $0.9 \%$ sodium chlorure solution. The solution quantity was used in 24 hours according to the patient weight (30 to $50 \mathrm{ml}$ per $\mathrm{Kg}$ ) and the aspect of thoracocentesis fluid. The skin was disinfected with the same antiseptic 10 minutes before the tube thoracostomy. In a case of allergy we replace the povidone iodine by the noxythioline.

Biological diagnosis of pleural empyema was based on Light's criteria for empyema: lactate dehydrogenase level of $>1000 \mathrm{IU} / \mathrm{L}, \mathrm{pH}$ of $<7.2$, glucose level of $<40 \mathrm{mg} / \mathrm{dL}$, and albumin level of $>30 \mathrm{~g} / \mathrm{L}$. The following data were obtained from the chart review: Sex, age, presenting symptoms (cough, fever) [temperature $>38^{\circ} \mathrm{C}$ ], weight loss, chest pain, shortness of breath, dehydration, duration of symptoms, co-morbidities (diabetes, HIV), and history of empyema. Details of the hospital admission on presentation included chest radiographic findings, previous antibiotic treatment, treatment procedures (chest tube, chest tube followed by antiseptic lavage irrigation), and pleural fluid isolated organisms were obtained. Bacterial culture was performed on pleural thoracocentesis fluid or blood specimens taken at admission for all children. The bacterial etiology of pleural empyema was assessed on the basis of a positive culture result from pleural fluid. Chest radiography was performed for all the patients at admission and after treatment started every week to determine the treatment efficacy. Patients with loculated empyema undergo chest echography to determine the location of the empyema in order to find out the convenient site of tube drainage with irrigation. Patients for group 1 and those for group 2 were started treatment with antibiotics empirically and later on changed according to bacterial culture and sensitivity of the antibiogram. CT scan was performed in patients who failed to tube thoracostomy procedure and were classified disease stage 2 or 3 according to the characteristic loculations and pleural peel found in late-stage disease. Stage repartition of pleural empyema was stage 1: exudative stage (acute); stage 2: fibinopurulent stage (transitional), stage 3: organizing stage (chronic). Patients with late stage with multiloculated empyema, broncho pleural fistula or pathological state of underlying lung were selected for video-assisted thoracoscopy surgery (VATS) or thoracotomy surgical procedure. Nutrition therapy was carried in regard of debilated children. Physiotherapy and breathing exercises were done following evacuation of the fluid to help in early re-expansion of the lung. Patients were discharged home once their condition became stable and were advised to come for follow up on monthly basis to assess their clinical and radiological improvement. Medical records of patients were retrospectively reviewed, including demographic information, laboratory data, and antimicrobial treatments.

The chest tube was removed when there was no drain or minimal drain $(<20 \mathrm{ml} /$ day $)$ for two consecutive days with a patent tube. The criteria for discharge for all patients were absence of fever for at least one week, chest tube removed, absence of tachypnea, and good oral acceptance. After discharge, all patients were followed up for a period of at least 6 weeks (visits on 1, 3 and 6 weeks). At each follow up, patients were assessed clinically, and subjected to chest radiograph. The primary outcomes were death or resolution of the empyema. Secondary outcomes addressed the length of time chest tubes were required, pain, hospital stay and any complications. Outcomes analysis with respect to treatment efficacy, hospital duration, chest tube duration, hospital costs, and need for subsequent procedures was analyzed and compared in the 2 groups.

The continuous data in this study are expressed as mean \pm standard deviation. Categorical variables were compared with the chi-square or Fisher's exact tests, and continuous variables were compared with unpaired t or Wilcoxon tests. Statistical analysis was performed with StatView (SAS Institute, Cary, NC, USA). P $<0.05$ was considered significant for all procedures.

\section{Results}

A total of 246 children aged from 1 to12 years were treated for empyema thoracis. In this study 152 patients (61.78\%) were 1 to 5 years and 94 patients (38.21\%) were 6 to 12 years of age. All the patients presented with complaints of cough, fever and breathlessness of variable durations. One hundred twenty three (50\%) children had history of pneumonia and treatment prior to development of empyema. One hundred seventy seven (72.08\%) children had right-sided and 69 (27.91\%) had left-sided empyema.

Chest X-ray done for all the patients within 48 hours of admission showed opacity varied from $20 \%$ to complete whitening of the hemithorax but we founded 56\% radiography image with more involvement of a pleural 
space. The ultrasonography showed loculations in $24 \%$ and pleural thickening in $8 \%$ of patients. The computerised tomography of chest was done after treatment failure for only 49 patients (19.91\%) and it showed loculation, collapse and pleural thickening in all. Patients in the two groups were similar with respect to preoperative demographics, clinical features and radiographic finding (Table 1). The pleural fluid aspirated was thick pus in 86\% in group 1 and 79\% in group 2; pleural fluid sugars were less than $40 \mathrm{mg} / \mathrm{dL}$ in $81 \%$ samples in the 2 groups. There is no statistical difference in the two groups in term of pleural fluids characteristics and blood parameters (Table 2).

In group one, $85.82 \%$ of children were successfully treated by drainage with antiseptic lavage-irrigation and $73.94 \%$ with tube thoracostomy alone in group 2 when associated with systemic antibiotics and other supportive measures. Eighteen (14.17\%) children failed to respond in group 1 and thirty one (26.05\%) in group 2 and needed VATS or decortications. VATS is performed in 16 patients with success and conversion to open thoracotomy for decortication is the setting of complex, chronic empyema in 33 patients. Most commonly isolated bacteria in pleural fluid were Echerichia coli, Staphylococcus aureus, Klebsiella pneumonia and Streptococcus pneumonia (Table 3). Blood culture was positive only for 14 patients in group 1 and for 21 patients in group 2 . Fever subsided in $56 \%$ of patients in group 1 within 3 days after admission with a median duration of 2 days and

Table 1. Baseline characteristics of patients with empyema finding at admission.

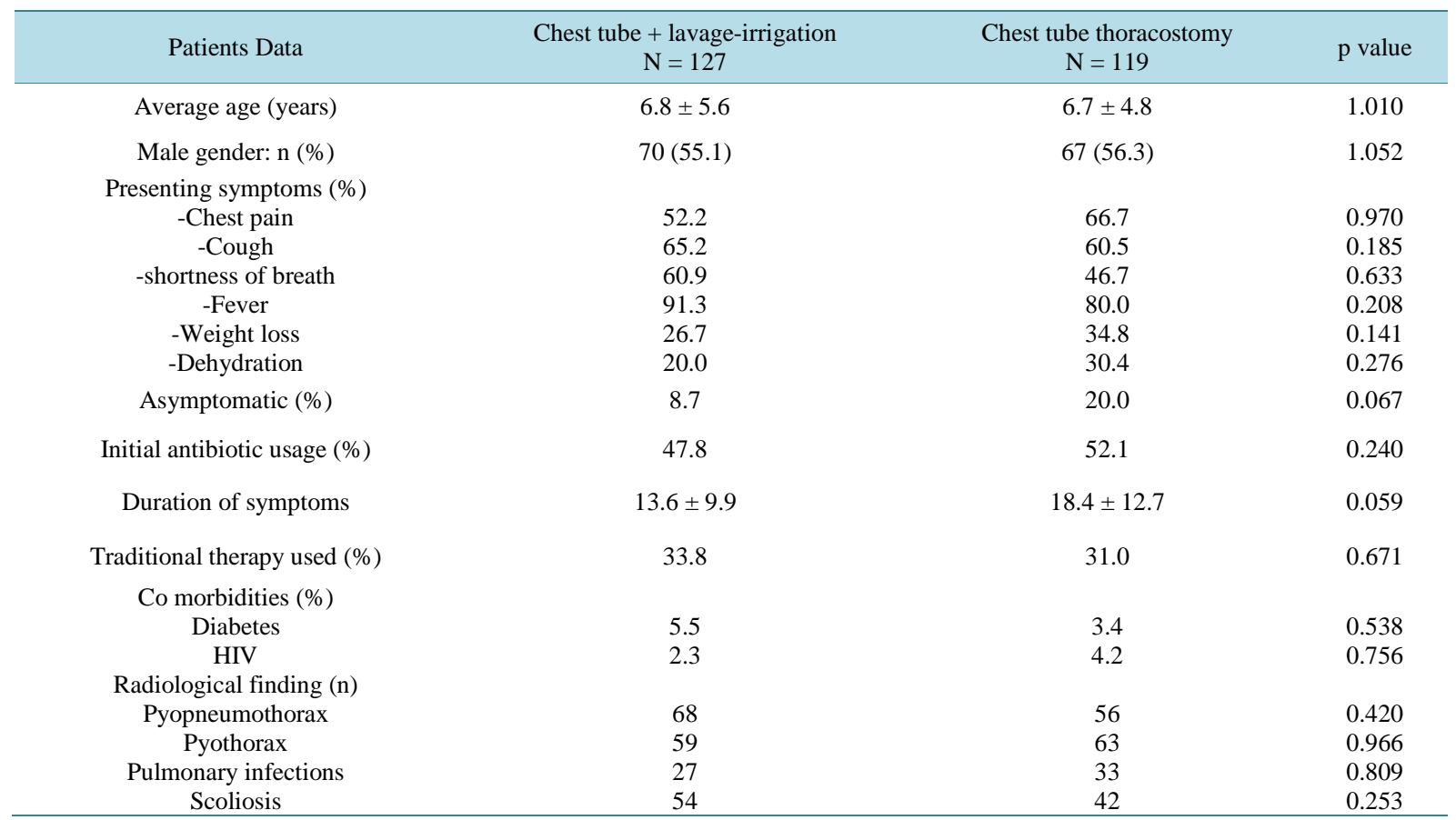

HIV: Human immunodeficiency virus.

Table 2. Distribution of patients according to hematology finding and pleural fluid characteristics.

\begin{tabular}{|c|c|c|c|}
\hline Characteristics & $\begin{array}{l}\text { Chest tube+lavage-irrigation } \\
\qquad \mathrm{N}=127\end{array}$ & $\begin{array}{l}\text { Chest tube thoracostomy } \\
\qquad \mathrm{N}=119\end{array}$ & $\mathrm{p}$ value \\
\hline \multicolumn{4}{|c|}{ Pleural fluid characteristics } \\
\hline Glucose (mg/dL) & $36.8 \pm 4.4$ & $31.00 \pm 11.6$ & 0.399 \\
\hline $\mathrm{WBC} / \mathrm{mm}^{3}$ & $4.4 \times 10^{4} \pm 4.6 \times 10^{4}$ & $3.5 \times 10^{4} \pm 4.7 \times 10^{4}$ & 0.130 \\
\hline $\mathrm{pH}$ & $7.3 \pm 0.0$ & $7.47 \pm 0.2$ & 0.566 \\
\hline $\mathrm{LDH}(\mathrm{U} / \mathrm{L})$ & $3.3 \times 10^{3} \pm 1.2 \times 10^{3}$ & $2.6 \times 10^{3} \pm 1.8 \times 10^{3}$ & 0.202 \\
\hline Protein (mg/dL) & $3.77 \pm 0.9$ & $4.40 \pm 0.8$ & 0.717 \\
\hline \multicolumn{4}{|l|}{ Blood parameters } \\
\hline $\mathrm{WBC} / \mathrm{mm}^{3}$ & $15,905 \pm 11,359.2$ & $13,660 \pm 6502.6$ & 0.803 \\
\hline Hemoglobin, g/l & $104 \pm 3.1$ & $9.8 \pm 2.7$ & 0.774 \\
\hline CRP, mg/dL & $30.7 \pm 1.2$ & $25.7 \pm 5.5$ & 0.492 \\
\hline
\end{tabular}

CRP: C-reactive protein; LDH: Lactate dehydrogenase; WBC: White blood cell count. 
only 41\% in group 2 within 3 days. The median duration of respiratory distress during hospital stay was 6 days. Significantly fewer postoperative complications occurred in the 2 groups in the following categories: atelectasis, partial lung expansion, prolonged air leak and septicemia but pulmonary embolism was found only in the group 2. There was no statistically significant difference in total complications and treatment failure among the 2 groups. One patient developed pericardial effusion secondary to empyema in group 1 (detected on echocardiography) that was minimal in amount and needed no surgical intervention. There are a significant difference in the length of hospital stay ( $p=0.022)$, duration of chest tubes in situ $(p=0.040)$, treatment coast $(p=0.015)$ and outcome of stage 2 empyema disease $(\mathrm{p}=0.037)$ between the 2 groups (Table 4$)$. Patients undergoing primary

Table 3. Distribution of patients according to organisms isolated.

\begin{tabular}{ccc}
\hline Bacteriology & Chest tube + lavage-irrigation & Chest tube thoracostomy \\
$\mathrm{N}=127$ & & $49(41.17)$ \\
Culture positive & $61(48.03)$ & 11 \\
Gram positive aerobic organisms & & 5 \\
Staphylococcus aureus & 14 & 4 \\
Staphylococcus epidermidis & 2 & 0 \\
Streptococcus pneumoniae & 5 & 1 \\
Streptococcus pyogenes & 2 & 3 \\
Enterococcus faecalis & 2 & 1 \\
Gram-Negative aerobic organism & & 4 \\
Haemophilus influenzae & 3 & 0 \\
Citrobacter freundii & 0 & 1 \\
Klebsiella pneumoniae & 8 & 7 \\
Anaerobic organisms & & 10 \\
Bacteroides melaninogenicus & 1 & 0 \\
Bacteroides vulgatus & 0 & 2 \\
Peptostreptococcus & 3 & \\
Echerichia coli & 19 & 1 \\
Fungal organism & 1 & \\
Aspergillus fumigatus & 1 & \\
Candida albicans & & \\
\hline
\end{tabular}

Table 4. Outcomes comparison between patients: chest tube antiseptic lavage-irrigation VS tube thoracostomy.

\begin{tabular}{cccc}
\hline Data & Chest tube + lavage-irrigation & Chest tube thoracostomy & N value \\
\hline Mortality n (\%) & $\mathrm{N}=127$ & $10(8.40)$ & 0.903 \\
Complications rate n (\%) & $7(5.51)$ & $42(35.29)$ & 0.833 \\
-Partial lung expansion/Atelectasis & $28(22.04)$ & 9 & 0.401 \\
-Air leak & 11 & 7 & 0.707 \\
-septicemia & 12 & 1 & 0.894 \\
-Pulmonary embolism & 4 & 0 & 0.907 \\
-Pericardial effusion & 0 & $31(26.05)$ & 0.250 \\
Treatment failure rate n (\%) & 1 & $22(18.48)$ & 0.075 \\
Stage 2 & $18(14.17)$ & $9(7.56)$ & $0.037^{*}$ \\
Stage 3 & $7(5.51)$ & $31.7 \pm 12.7$ & 0.110 \\
Length of stay (days) & $11(8.66)$ & $24.5 \pm 6.7$ & $0.022^{*}$ \\
Chest tube in situ (days) & $19.4 \pm 9.7$ & $12.8 \pm 21.3$ & $0.040^{*}$ \\
Antibiotics used (days) & $10.1 \pm 3.4$ & & 0.688 \\
Subsequent procedures need n (\%) & $13.6 \pm 19.9$ & $96(80.67)$ & 0.063 \\
-Aspiration & & $110(92.43)$ & 1.046 \\
- Respiratory kinesitherapy & $45(35.43)$ & $93(78.15)$ & 0.907 \\
Postoperative pain n (\%) & $119(93.70)$ & $53(44.53)$ & 0.451 \\
Re-drainage n (\%) & $85(66.92)$ & $19(15.96)$ & 0.866 \\
Hospital cost (USD) & $34(26.77)$ & $258.5 \pm 7.5$ & $0.015^{*}$ \\
Recovery times (days) & $12(9.44)$ & $38.4 \pm 9.7$ & 0.052 \\
\hline
\end{tabular}


chest tube drainage alone had a significantly longer hospital stay and duration of chest tube in situ than the patients with antiseptic lavage-irrigation system. Moreover, patients undergoing chest tube drainage with antiseptic lavage-irrigation had significant lower total hospital charges. Each group suffered one mortality ( $p=$ not significant). The mortality rate in the study was $6.91 \%$.

\section{Discussion}

The mainstay of pleural empyema treatment is the control of ongoing infection and the prevention of recurrent infection and late restriction [6]. Criteria for selection of therapy likely evolved with time and socio-economical conditions; the care of empyema among children has progressed in the past 20 years. Poverty and low immunity in younger age group may be the contributing factor to develop empyema thoracic. Children are often referred late to the surgeon in a malnourished state [7]. The families usually end-up spending all their meager resources on consultations and prolonged courses of expensive broad spectrum antibiotics far more than the cost of adequate treatment in a hospital. Misthos et al. [8] in their study reported fever as the most common presenting symptom (88\%) followed by cough (73\%). We also observed fever as the frequent symptom (85.77\%) followed by cough $(61,78)$. Opacity was found on more than $56 \%$ of a pleural space on $x$-ray chest which emphasizes the role of X-ray chest in patients presenting with cough, fever, and dyspnea. Treatment of thoracic empyema includes three basic principles: 1) drainage of complicated pleural effusion; 2) full expansion of the underlying lung and 3) elimination of the pleuropulmonary infection with antimicrobial agents [9]. There are different types of treatments for different stages of the empyema thoracic. The decision of the type of treatment is usually empiric, sometimes guided by the failure of another treatment modality [2]. Mandal et al. [10] in their study reported 62\% success rate for closed thoracostomy, our cure rate (73.94\%) with tube tube thoracostomy was almost the same. Interestingly; results of chest tube drainage with antiseptic lavage-irrigation system are different (85.82\%) and leads to improved outcomes in early empyema thoracis. Chest tube drainage combined with antiseptic lavage-irrigation had a significantly higher primary treatment success than chest tube thoracostomy alone in patients with stage 1 or early stage 2 empyema thorasis. Patients undergoing this procedure spent less time in hospital comparing to the tube thoracostomy alone $(p=0.022)$. Although this nonoperative therapy may be successful for the treatment of early empyema stage 1 and stage 2 (uni-loculated empyema) diseases. When chest tube was placed with the antiseptic lavage-irrigation catheter into the early loculated empyema space under chest echography, pulmonary expansion is usually obtained in childreen. The characteristic loculations and pleural peel found in late-stage disease make adequate drainage of the infected pleural space and expansion of the lung through simple drainage more difficult. However, incomplete drainage of the pleural space with persistent signs of infection should prompt surgical intervention [11]. The advent of video-assisted thoracic surgery (VATS) for the management of fibrinopurulent stage 2 empyema has shown rewarding results in several reports [12] [13]. VATS is used successfully for management of stage 2 empyema in $6.50 \%$ of our patients. Treatment failure in all groups using antiseptic lavage irrigation or not represented patient with late stage disease (stage 2 or 3 ) and patients undergoing operative therapy presumed to have advanced disease. Chronic cases and complications such as broncho-pleural fistula, a lesion of the lung, recurrent severe hemoptysis, fibrothorax may requires aggressive intervention like wedge resection or decortications [14]. In this study a few patients of stage 2 or 3 pleural empyema with multiloculated empyema, broncho pleural fistula or pathological state of underlying lung who fail to the measures need complex surgical procedures. There is difference between the 2 groups concerning the treatment failure rate for stage 2 empyema disease. Drainage with antiseptic lavage-irrigation seems to improve outcome in the early stage 2 children empyema thorasis. Properly sited intercostals tube drainage associated with antiseptic lavage-irrigation system during the early stage of pathology could avoid surgery or morbid complication in developing country. A properly managed case of empyema would prevent serious complications that may later require major surgical treatment. However the success of any intervention depends on the status of the underlying lung pathology and the proper usage of antibiotics. Fibrinolytics (streptokinase and urokinase) have become part of the armamentarium for treatment of pediatric empyema [15]. The inclusion of fibrinolytics to chest tube drainage creates a potential limitation for comparisons of this method with another nonoperative treatment. Given the comparable effectiveness of fibrinolytics with respect to nonoperative therapy it remains a significant difference in utilization of resources. In most of the pediatric studies reviewed, fibrinolytic therapy was used for children who failed to exhibit improvement with nonoperative therapy alone. In fact, salvage fibrinolytic therapy may cause worsening of intrapleural loculations and increase the difficulty of the VATS proce- 
dure [16]. However, fibrinolytics were not used in this study because of the treatment unavailability in our context.

Rapid diagnosis of the causal agent is required for effective treatment and a good outcome [7]. Staphylococcus aureus is the most commonly retrieved organism [10] but in this study we most retrieved Escherichia coli followed by Staphylococcus aureus. The classic microbiological technique for diagnosis of pleural empyema is standard microbiological culture and direct examination of pleural fluid [17] [18]. Culture is essential because antimicrobial susceptibility can be determined. However, cultures are slow and can have false-negative results because of small sample volume, previous antibiotic therapy, or unsatisfactory conditions of transport and storage, which can impair the viability of pathogens, particularly Streptococcus pneumoniae. It is possible to improve diagnostic sensitivity by using broad-range 16 S rDNA PCR and sequencing [19], which permit detection of potentially all bacterial etiologies, or by using amplification of species-specific genes, such as the pneumolysin gene (ply) of S. pneumoniae [18] [20]. Given the economic context, these techniques are difficult to be carried out in a poor-resource country. The cultures are steriles in 30\% - 50\% of cases dues to antibiotics [21]. In our study cultures are steriles in (44.71\%) of cases. Previous antibiotic therapies as well as unsatisfactory conditions of transport and storage were involved. Chest tube drainage and antiseptic lavage-irrigation used routinely in our context as a first-line measure in empyema thoracis management in children is a safe and relatively atraumatic procedure. The method does not exclude the use of any subsequent surgical measure, and provides optimal time to improve the condition of debilitated patients so that when it comes to perform surgery, they may tolerate more aggressive surgical procedures.

Meta analysis comparing operative and non-operative procedures has concluded that conservative management leads to recovery in more than $76 \%$ of the patients [22]. However, it needs to be emphasised that a less invasive primary operative procedure like drainage with lavage-irrigation has the potential to interrupt the progression of empyema, decrease discomfort associated with prolonged thoracostomy tube usage and reduce the total duration of hospital stay and chest tubes in situ. Further, the role of these interventions is better defined in patients with early empyema in the pleural cavity. The study showed a good pleural recovery in children of group 1 with stage 1 or 2 . Clinically relevant but not statistically significant differences in total complications rates $(22.04 \%$ vs $35.29 \%)$ and total treatment failure rate $(14.17 \%$ vs $26.05 \%)$ also favored the group 1 . Scoliosis detected on the chest radiography in the follow up resolved in all children without further intervention in 2 16 months time [7]. No treatment for scoliosis was conducted in our patients. The patients who died were severely debilitated with severe denutrition. Their deaths were not related to the technique, which was well tolerated in all cases.

\section{Conclusion}

As a primary treatment strategy in children empyema thoracis; it seems that chest tube drainage with chest catheter antiseptic lavage-irrigation is superior to chest tube thoracostomy alone in terms of duration of chest tubes in situ, length of hospital stay, treatment cost and outcome of stage 2 empyema disease. The antiseptic lavageirrigation method combine with physiotherapy, antibiotics and nutrition treatment gives good results and greater patient acceptance especially in resource-poor settings. However more studies are needed to draw conclusions.

\section{References}

[1] Satish, B., Bunker, M. and Seddon, P. (2003) Management of Thoracic Empyema in Childhood: Does the Pleural Thickening Matter? Archives of Disease in Childhood, 88, 918-921. http://dx.doi.org/10.1136/adc.88.10.918

[2] Yilmaz, E., Dogan, Y., Aydinoglu, A.H., Gurgoze, M.K. and Aygun, D. (2002) Parapneumonic Empyema in Children: Conservative Approach. The Turkish Journal of Pediatrics, 44, 134-138.

[3] Long, A.M., Smith-Williams, J., Mayell, S., Couriel, J., Jones, M.O. and Losty, P.D. (2015) “Less May Be Best”Pediatric Parapneumonic Effusion and Empyema Management: Lessons from a UK Center. Journal of Pediatric Surgery, 12, 455-458.

[4] Yao, C.T., Wu, J.M., Liu, C.C., Wu, M.H., Chuang, H.Y. and Wang, J.N. (2004) Treatment of Complicated Parapneumonic Pleural Effusion with Intrapleural Streptokinase in Children. Chest, 125, 566-571. http://dx.doi.org/10.1378/chest.125.2.566

[5] Light, R.W. (1995) A New Classification of Parapneumonic Effusions and Empyema. Chest, 108, $299-301$. http://dx.doi.org/10.1378/chest.108.2.299 
[6] Rzyman, W., Skokowski, J., Romanowicz, G., Lass, P. and Dziadziuszko, R. (2002) Decortications in Chronic Pleural Empyema-Effect on Lung Function. European Journal Cardio-Thoracic Surgery, 21, 502-507. http://dx.doi.org/10.1016/S1010-7940(01)01167-8

[7] Goyal, V., Kumar, A., Gupta, M., Sandhu, H.P. and Dhir, S. (2014) Empyema Thoracis in Children: Still a Challenge in Developing Countries. African Journal of Paediatric Surgery, 11, 206-210. http://dx.doi.org/10.4103/0189-6725.137326

[8] Misthos, P., Sepsas, E., Konstantinou, M., Athanassiadi, K., Skottis, I. and Lioulias, A. (2005) Early Use of Intrapleural Fibrinolytics in the Management of Postpneumonic Empyema. A Prospective Study. European Journal CardioThoracic Surgery, 28, 599-603. http://dx.doi.org/10.1016/j.ejcts.2005.07.005

[9] Huang, H.C., Chang, H.Y., Chen, C.W. and Lee, C.H. (1999) Predicting Factors for Outcome of Tube Thoracostomy in Complicated Parapneumonic Effusion or Empyema. Chest, 115, 751-756. http://dx.doi.org/10.1378/chest.115.3.751

[10] Mandal, A.K., Thadepalli, H., Mandal, A.K. and Chetipally, U. (1998) Outcome of Primary Emypema Thoracis: Therapeutic and Microbiologic Aspects. The Annals of Thoracic Surgery, 66, 1782-1786. http://dx.doi.org/10.1016/S0003-4975(98)00601-8

[11] Ris, H.B., Pezzetta, E., Krueger, T. and Lardinois, D. (2004) Surgical Treatment of Pleural Infections: The Surgeon's Point of View. European Respiratory Monograph, 29, 181-188. http://dx.doi.org/10.1183/1025448x.00029012

[12] Waller, D.A. and Rengarajan, A. (2001) Thoracoscopic Decortication: A Role for Video-Assisted Surgery in Chronic Postpneumonic Pleural Empyema. The Annals of Thoracic Surgery, 71, 1813-1816. http://dx.doi.org/10.1016/S0003-4975(01)02471-7

[13] Roberts, J.R. (2003) Minimally Invasive Surgery in the Treatment of Empyema: Intraoperative Decision Making. The Annals of Thoracic Surgery, 76, 225-230. http://dx.doi.org/10.1016/S0003-4975(03)00025-0

[14] Scarci, M., Abah, U. and Solli, P. (2015) EACTS Expert Consensus Statement for Surgical Management of Pleural Empyema. European Journal of Cardio-Thoracic Surgery, 48, 642-653. http://dx.doi.org/10.1093/ejcts/ezv272

[15] Bose, K., Saha, S., Mridha, D., Das, K., Mondal, P. and Das, I. (2015) Analysis of Outcome of Intraplueral Streptokinase in Pediatric Empyema Thoracis Even in Advanced Stages: A Prospective Study. Iranian Journal of Pediatrics, 25, e3154. http://dx.doi.org/10.5812/ijp.3154

[16] Jaffe, A. and Cohen, G. (2003) Thoracic Empyema. Archives of Disease in Childhood, 88, 839-841. http://dx.doi.org/10.1136/adc.88.10.839

[17] Cohen, G., Hjortdal, V., Ricci, M., et al. (2003) Primary Thoracoscopic Treatment of Empyema in Children. Journal of Thoracic and Cardiovascular Surgery, 125, 79-83. http://dx.doi.org/10.1067/mtc.2003.88

[18] Doski, J.J., Lou, D., Hicks, B.A., et al. (2000) Management of Parapneumonic Collections in Infants and Children. Journal of Pediatric Surgery, 35, 265-268. http://dx.doi.org/10.1016/S0022-3468(00)90022-8

[19] Alexiou, C., Goyal, A., Firmin, R.K. and Hickey, M.S. (2003) Is Open Thoracotomy Still a Good Treatment Option for the Management of Empyema in Children? The Annals of Thoracic Surgery, 76, 1854-1858. http://dx.doi.org/10.1016/S0003-4975(03)01076-2

[20] Hilliard, T.N., Henderson, A.J. and Langton Hewer, S.C. (2003) Management of Parapneumonic Effusion and Empyema. Archives of Disease in Childhood, 88, 915-917. http://dx.doi.org/10.1136/adc.88.10.915

[21] Saleem, A.F., Shaikh, A.S., Khan, R.S., Khan, F., Faruque, A.V. and Khan, M.A. (2014) Empyema Thoracis in Children: Clinical Presentation, Management and Complications. Journal of the College of Physicians and Surgeons Pakistan, 24, 573-576.

[22] Avansino, J.R., Goldman, B., Sawin, R.S. and Flum, D.R. (2005) Primary Operative versus Nonoperative Therapy for Pediatric Empyema: A Meta-Analysis. Pediatrics, 115, 1652-1659. http://dx.doi.org/10.1542/peds.2004-1405 